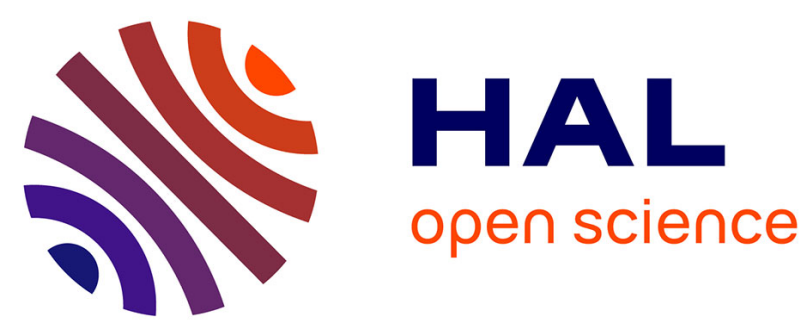

\title{
In vivo optogenetic stimulation of the primate retina activates the visual cortex after long-term transduction
}

Antoine Chaffiol, Matthieu Provansal, Corentin Joffrois, Kévin Blaize, Guillaume Labernede, Ruben Goulet, Emma Burban, Elena Brazhnikova, Jens Duebel, Pierre Pouget, et al.

\section{To cite this version:}

Antoine Chaffiol, Matthieu Provansal, Corentin Joffrois, Kévin Blaize, Guillaume Labernede, et al.. In vivo optogenetic stimulation of the primate retina activates the visual cortex after longterm transduction. Molecular Therapy - Methods and Clinical Development, 2022, 24, pp.1 - 10. 10.1016/j.omtm.2021.11.009 . hal-03512112

\section{HAL Id: hal-03512112 \\ https://hal.sorbonne-universite.fr/hal-03512112}

Submitted on 5 Jan 2022

HAL is a multi-disciplinary open access archive for the deposit and dissemination of scientific research documents, whether they are published or not. The documents may come from teaching and research institutions in France or abroad, or from public or private research centers.
L'archive ouverte pluridisciplinaire HAL, est destinée au dépôt et à la diffusion de documents scientifiques de niveau recherche, publiés ou non, émanant des établissements d'enseignement et de recherche français ou étrangers, des laboratoires publics ou privés. 


\title{
In vivo optogenetic stimulation of the primate retina activates the visual cortex after long-term transduction
}

\author{
Antoine Chaffiol, ${ }^{1}$ Matthieu Provansal, ${ }^{1}$ Corentin Joffrois, ${ }^{1}$ Kévin Blaize, ${ }^{2}$ Guillaume Labernede, ${ }^{1}$ Ruben Goulet, ${ }^{1}$ \\ Emma Burban, ${ }^{1}$ Elena Brazhnikova, ${ }^{1}$ Jens Duebel, ${ }^{1,4}$ Pierre Pouget, ${ }^{3}$ José Alain Sahel, ${ }^{1,5}$ Serge Picaud, ${ }^{1}$ \\ Fabrice Arcizet, ${ }^{1,6}$ and Gregory Gauvain ${ }^{1,6}$
}

\begin{abstract}
${ }^{1}$ Sorbonne Université, Inserm, CNRS, Institut de la Vision, 75012 Paris, France; ${ }^{2}$ Institut de Neurosciences de la Timone, UMR 7289 Centre National de la Recherche Scientifique and Aix-Marseille Université, 13385 Marseille Cedex 05, France; ${ }^{3}$ INSERM 1127, CNRS 7225, Institut du Cerveau et de la Moelle Épinière, Sorbonne Université, 75013 Paris, France; ${ }^{4}$ Department of Ophthalmology, University Medical Center Göttingen, Göttingen, Germany; ${ }^{5}$ Department of Ophthalmology, The University of Pittsburgh School of Medicine, Pittsburgh, PA 15213, USA
\end{abstract}

Over the last 15 years, optogenetics has changed fundamental research in neuroscience and is now reaching toward therapeutic applications. Vision restoration strategies using optogenetics are now at the forefront of these new clinical opportunities. But applications to human patients suffering from retinal diseases leading to blindness raise important concerns on the long-term functional expression of optogenes and the efficient signal transmission to higher visual centers. Here, we demonstrate in non-human primates continued expression and functionality at the retina level $\sim 20$ months after delivery of our construct. We also performed in vivo recordings of visually evoked potentials in the primary visual cortex of anesthetized animals. Using synaptic blockers, we isolated the in vivo cortical activation resulting from the direct optogenetic stimulation of primate retina. In conclusion, our work indicates long-term transgene expression and transmission of the signal generated in the macaque retina to the visual cortex, two important features for future clinical applications.

\section{INTRODUCTION}

Repairing sensory impairments has always been an overarching goal in medicine. In the particular case of vision loss, considerable progress has been achieved in recent years through the development of various therapeutic strategies, such as retinal prostheses, ${ }^{1-4}$ stem cell transplantation, $^{5-8}$ and optogenetic therapies. ${ }^{9-21}$ All these approaches aspire to restore retinal visual information. Microbial opsin-based optogenetics is one of the most promising of these approaches. It involves the expression of light-sensitive ion channels in preserved inner retinal neurons, restoring the intrinsic light sensitivity of the pathological retina in several types of ocular disease.

In inherited forms of retinal degeneration, such as retinitis pigmentosa (RP), the retinal degeneration is progressive, beginning with retinal pigment epithelium (RPE) or photoreceptors dysfunction, and possibly causing complete blindness. ${ }^{22}$ The choice of target cell type in the retinal circuit should take into account the potential for translation into clinical applications and uses in patients. The accessibility of the targeted cell population and the maintenance of its structure and integrity after the onset of retinal degeneration are key features. Since the first use of optogenetics to restore vision in blind mice through the expression of channelrhodopsin-2 (Chr2) in retinal ganglion cells (RGCs), ${ }^{10}$ many other studies have been conducted, targeting different cell types in the retina: photoreceptors, ${ }^{14,23}$ bipolar cells, ${ }^{18,19}$ or RGCs. ${ }^{9,10,15,17,20}$ Importantly, in diseases such as age-related macular degeneration and RP, the RGCs remain well-preserved during the process of retinal degeneration, even at late stages of the disease, after the death of the photoreceptors. ${ }^{24}$ Various models, including rodents, non-human primates (NHPs), postmortem human retina, and human induced pluripotent stem cells, have been used for investigations of optogenetic approaches, with promising results. ${ }^{7,10,17,23}$

Primates are a pertinent animal model for preclinical validation of optogenetic therapeutic approaches ${ }^{25}$ because they share essential anatomical features and a similar organization of visual pathways with humans. However, few studies to date have used this animal model for investigations of the potential of optogenetic therapy for the retina. For example, several opsins targeting RGCs have been tested in ex vivo preparations, including the microbial opsin channelrhodopsin-2 in marmosets ${ }^{9}$ and CatCh, ReaChr, and ChR-tdT in macaques. ${ }^{11,15,17}$ All these opsins were found to be functional in

\footnotetext{
Received 21 September 2021; accepted 19 November 2021; https://doi.org/10.1016/j.omtm.2021.11.009.

${ }^{6}$ These authors contributed equally

Correspondence: Antoine Chaffiol, $\mathrm{PhD}$, Sorbonne Université, Inserm, CNRS, Institut de la Vision, 75012 Paris, France.

E-mail: antoine.chaffiol@inserm.fr

Correspondence: Fabrice Arcizet, $\mathrm{PhD}$, Sorbonne Université, Inserm, CNRS, Institut de la Vision, 75012 Paris, France.

E-mail: fabrice.arcizet@inserm.fi
} 
RGCs. Furthermore, the optogenetic responses of RGCs in vivo have been recorded with calcium imaging after photoablation of the photoreceptors in the macaque foveal region. ${ }^{20}$ However, none of these studies has shown that the propagation of optogenetic activation from RGCs to higher visual centers, such as the primary visual cortex, was possible. Such experiments on retinal optogenetic approaches have been performed only in rodents and have shown that the optogenetic activation of transduced retinae induces specific visual evoked responses (VEPs) in the visual cortex. ${ }^{14,15,18,26,27}$ Moreover, specific cortical responses were recorded following the activation of RGCs by photovoltaic subretinal implants in rats. ${ }^{28} \mathrm{~A}$ recent study on primates fitted with subretinal implants showed that these prostheses induced some behavioral responses. ${ }^{2}$ However, to our knowledge, no study to date has demonstrated the transmission of information to higher visual areas following activation of the optogenetically transduced retina in primates. But a recent study in human patients with Leber congenital amaurosis associated with RPE65 gene deficiency and treated with subretinal injection of an adeno-associated virus (AAV) vector has shown cortical activation along visual pathways during fRMI analysis. ${ }^{29}$

Using this approach to optogenetic therapy, we targeted the RGCs in primate retinas through the in vivo expression of an ectopic light-sensitive ion channel, ChrimsonR, coupled to the fluorescent reporter tdTomato. ${ }^{17}$ The possible application of this strategy to blind patients suffering from retinal dystrophies raises important concerns about long-term functional expression to ensure efficient signal transmission to higher brain centers ${ }^{30}$ (i.e., the visual cortex). We previously showed that the transduced retina 2 or 6 months after injection displays a high degree of spatiotemporal resolution ex vivo, compatible with the perception of highly dynamic visual scenes at light levels suitable for use in humans. ${ }^{17}$ Here, we demonstrate, in NHPs, sustained functional efficacy $\sim 20$ months after the delivery of an AAV2.7m8ChrimsonR-tdTomato vector similar to that currently undergoing clinical evaluation. Our results reveal a persistence of expression in the perifovea, mediating information transfer to higher brain centers. Indeed, we recorded visual evoked potentials (VEPs) in the primary visual cortex of anesthetized primates in response to optogenetic retinal activation. We used an intravitreal injection of synaptic blockers to isolate the cortical component resulting from the in vivo optogenetic stimulation of primate RGCs. Our findings demonstrate the long-term functional efficacy of optogenetic therapy to restore information transfer from the retina to the brain in vivo.

\section{RESULTS}

The experiments were conducted on three monkeys (Macaca fascicularis), each of them receiving, in one eye, a single intra-vitreal injection of AAV2.7m8-ChrR-tdT at a dose of $5 \times 10^{11} \mathrm{vg} / \mathrm{eye}$, the other eye being kept as a control. More than 20 months later, we performed in vivo cortical experiments to record VEPs to the retinal optogenetic stimulation. Subsequently, $\sim 24 \mathrm{~h}$ after the euthanasia, we measured directly optogenetic responses on ex vivo retinal foveal explants. For every eye treated with ChrR-tdT, half of the retina was used for single-cell RGC recordings and two-photon imaging, whereas the other half was used for multi-electrode array (MEA) RGC population recordings and later histology.

The long-term functional expression of ChrimsonR-tdTomato (ChRtdT) was assessed in the primate retina by ex vivo retinal recordings in the presence of glutamate receptor antagonists (see materials and methods), which were added to the bath solution to suppress any natural light response. Live epifluorescence images revealed a high density of transfected cells localized in the perifoveolar region, forming a torus shape (Figure 1A), mostly in the retinal ganglion cell layer (Figures $1 \mathrm{~B}$ and Video S1). On some of these transfected RGCs, using two-photon live imaging, we observed small dendritic arbors, suggesting that at least some foveal midget RGCs responsible for highacuity vision $^{31}$ were expressing ChR-tdT (Video S1). Light sensitivity and temporal dynamics were measured, at the single-cell level, with the two-photon guided patch-clamp technique, on ChR-tdT-positive cells (Figure 1C). We found that the mean normalized photocurrent response increased significantly with increasing light intensity (Figure $1 \mathrm{D}$, top), reaching a value of $143.6 \pm 47.4 \mathrm{pA}(n=12)$ at a light intensity of $3 \times 10^{17}$ photons. $\mathrm{cm}^{-2} . \mathrm{s}^{-1}$. In the loose-patch recording configuration, firing rate displayed a similar dependence on light level $(99.03 \pm 7.59 \mathrm{~Hz}, n=32$ cells), also peaking at a light intensity of $3 \mathrm{x}$ $10^{17}$ photons. $\mathrm{cm}^{-2} \cdot \mathrm{s}^{-1}$ (Figure 1E, right). We then showed that the highest frequency responses were obtained for light stimuli at $575 \mathrm{~nm}$ (Figure 1F), corresponding to the excitation peak of ChrimsonR $(590 \mathrm{~nm}){ }^{32}$ We obtained reliable firing bursts with fast dynamics during the measurement of RGC responses to stimuli of increasing durations (20 ms-4 s, $n=9$; Figure $1 \mathrm{G})$ or various flicker frequencies (10 repeats in full duty cycle) up to $28 \mathrm{~Hz}(n=9$; Figure $1 \mathrm{H})$. These results demonstrate the ability of these engineered cells to follow and resolve short, long, and fast light stimulations accurately up to frequencies very similar to the video-rate frequencies $(\sim 25 \mathrm{~Hz})$ required for fluid movement perception and compatible with the limits for flicker perception reported for humans. ${ }^{33}$

In parallel, we used MEA recordings to investigate the responsiveness of the cell population in the transfected area (Figures 2 and S2). A large proportion of the perifovea contained a high density of ChRtdT-expressing cells, as indicated by counting tdTomato-positive cells on projections of confocal stacks (Figures $2 \mathrm{~A}$ and $2 \mathrm{~B}$ ). The MEA chip (Figure 2C, top left) covered a large area of the hemifoveal retina flat mounts, making it possible to take recordings for a large proportion of the transfected RGCs (Figure 2C, bottom left; Figure S2A). Figures 2C (right) and S2C show the global recorded activity for RGCs, represented as the firing rate of 256 sites over a period of $4 \mathrm{~s}$ in response to a 2 -s flash of light. We performed 10 recordings in total, at a wavelength of $595 \mathrm{~nm}$ and a light intensity of $7 \times 10^{16}$ photons. $\mathrm{cm}^{2} \cdot \mathrm{s}^{-1}$ (Figure 2C, right). A large proportion of the recording sites were responsive, and the response observed was correlated to the degree of optogene expression, as determined by measuring tdTomato reporter fluorescence. Overall, in the two retinas for which we were able to obtain spontaneous activity, $45.5 \%$ of all active recording sites were also responsive to light stimulation (172 of 378 active sites; Figure 2D). We performed MEA recordings as a function of light 
A
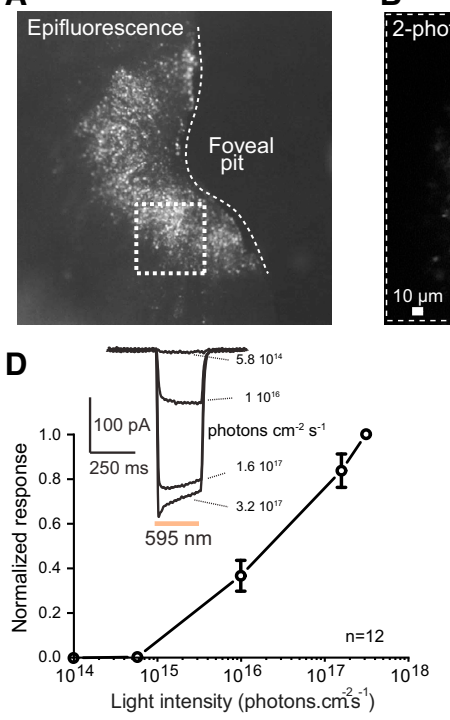

G

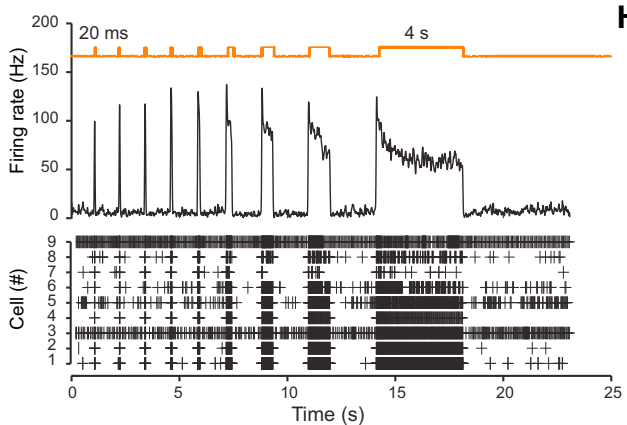

H
B

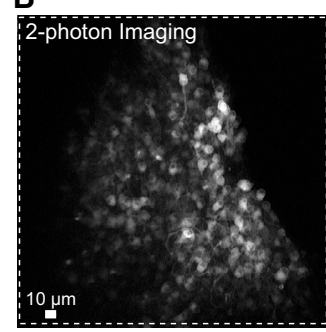

E

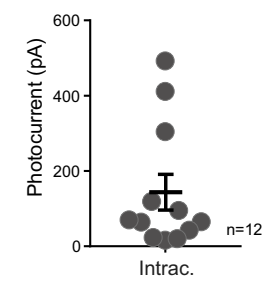

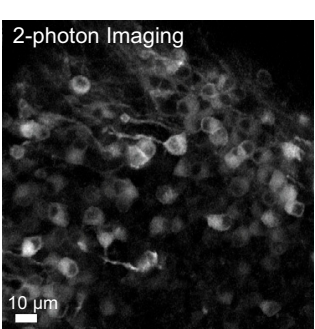

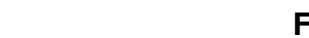

$\mathbf{F}$

C
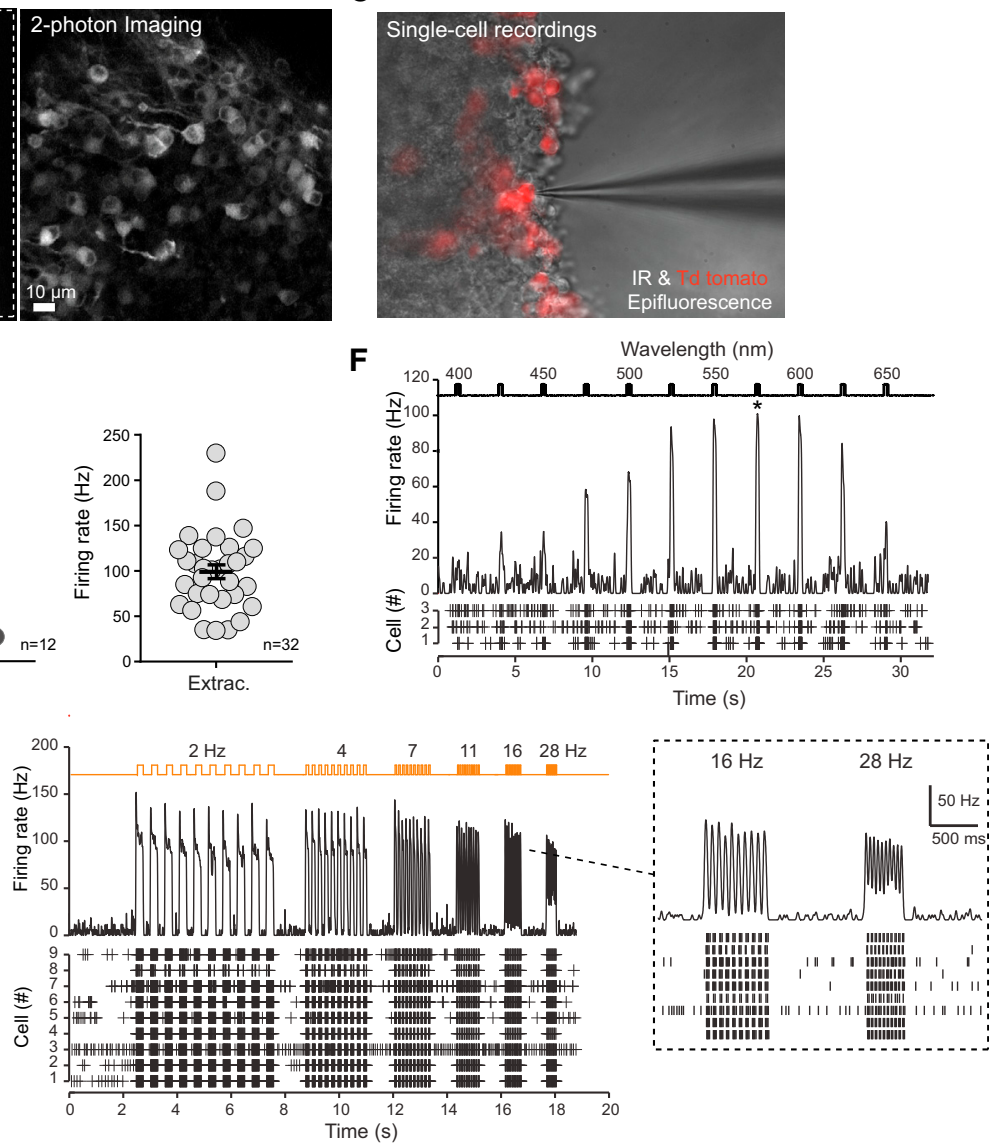

Figure 1. Long-lasting expression of the optogene in the macaque perifoveal ring and two-photon guided single-cell recordings

(A) Epifluorescence image of an ex vivo primate hemifovea expressing tdTomato-ChrimsonR (perifoveal ring) 20 months after the injection of AAV2.7m8-ChR-tdT at a dose of $5 \times 10^{11} \mathrm{vg} / \mathrm{eye}$. (B) Examples of two-photon images of tdTomato-positive cells in the retinal ganglion cell layer; the left panel represents the retinal area taken in the dotted square shown in (A), while the right panel shows an example from another transduced retina at higher magnification. (C) Combined infrared and epifluorescence image of tdTomato-expressing fluorescent cells during single-cell recordings with a glass electrode (patch-clamp or extracellular recordings). (D) Photocurrent response as a function of the intensity of the light stimulus in transfected retinal ganglion cells 20 months post injection, and comparison with earlier time points. Example of photocurrents (top) recorded in cells stimulated with light at $595 \mathrm{~nm}$ for $250 \mathrm{~ms}$ at intensities of $5.8 \times 10^{14}$ to $3.2 \times 10^{17}$ photons. $\mathrm{cm}^{2} . \mathrm{s}^{-1}$. Normalized intensity curve (bottom) for 12 patched cells. (E) Peak responses for photocurrent (left, patch-clamp data, $n=12$ cells) and firing rate (right, extracellular recordings, $n=32$ cells) recorded in RGCs for a light stimulus at a wavelength of $595 \mathrm{~nm}$ and an intensity of $3.2 \times 10^{17}$ photons. $\mathrm{cm}^{2} . \mathrm{s}^{-1}$. Long horizontal black lines represent the mean $\pm \mathrm{SEM}$. (F) Spectral tuning. RGC response (raster plot and firing rate) as a function of the stimulus wavelength tested, for wavelengths between 400 and $650 \mathrm{~nm}$ ( $n=3$ cells). The peak response (asterisk) was observed at $575 \mathrm{~nm}$. The black curve represents the mean cell response. (G) RGC response as a function of stimulus duration. Raster plot (bottom) for nine cells and firing rate (top), with stimuli of increasing duration, from $20 \mathrm{~ms}$ to $4 \mathrm{~s}$. The black curve represents the mean cell response. (H) Temporal resolution. RGC activity as a function of stimulation frequency, represented as a raster plot (bottom) and firing rate (top), for 10 flicker repeats at frequencies from $2 \mathrm{~Hz}$ to $28 \mathrm{~Hz}$ ( $n=9$ cells). The black curve represents the mean cell response.

intensity (Figure 2E). The results obtained were very similar to those obtained with the single-cell technique (Figures $1 \mathrm{D}$ and $1 \mathrm{E}$ ). Indeed, the threshold light intensity for a response was found to be $9 \times 10^{15}$ photons. $\mathrm{cm}^{2} . \mathrm{s}^{-1}$. We then analyzed MEA responses as a function of stimulus duration (Figure $2 \mathrm{~F}$ ), at an intensity of $7 \times 10^{16}$ photo$\mathrm{ns} . \mathrm{cm}^{2} . \mathrm{s}^{-1}$. In the two retinas tested, cells responded to stimuli with a duration of at least $5 \mathrm{~ms}$, but not to stimuli of shorter duration.

In living animals, we then investigated whether the optogenetic stimulation of these transfected RGCs could activate the visual pathway in vivo. To this end, we recorded VEPs in response to different visual stimuli presented to the anesthetized animals during short-term experiments (Figure $3 \mathrm{~A}$ ). In this way, we were able to compare VEP responses before and after the intravitreal injection of synaptic blockers (2,3-piperidine dicarboxylic acid [PDA] and L-(+)-2-amino-4-phosphonobutyric acid [L-AP4]) into both eyes (Figure 3B) to block natural retinal responses to light. ${ }^{34}$ The kinetic of action for the two blockers was first determined by a control electro-retinogram (ERG) experiment on a naive animal (Figure S3) that showed a complete suppression of the ERG's b-wave in 40 min that lasted $24 \mathrm{~h}$. 

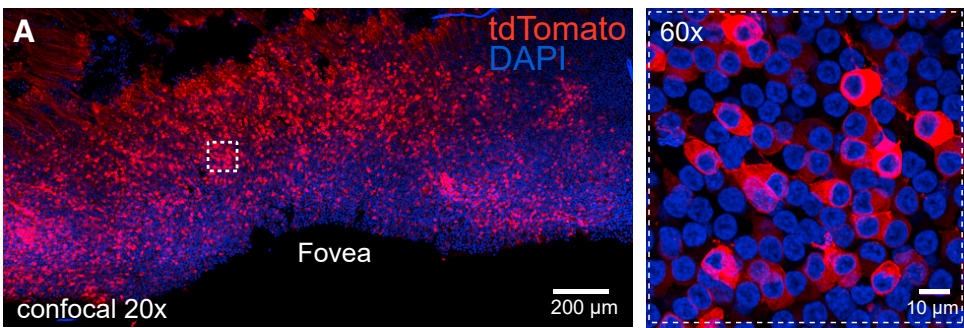

C

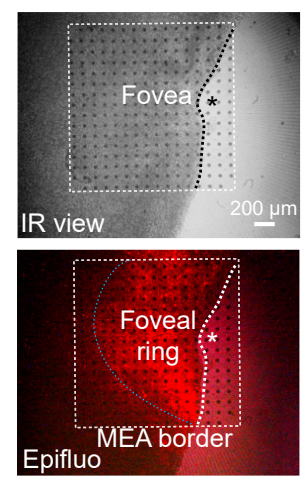

MEA recordings
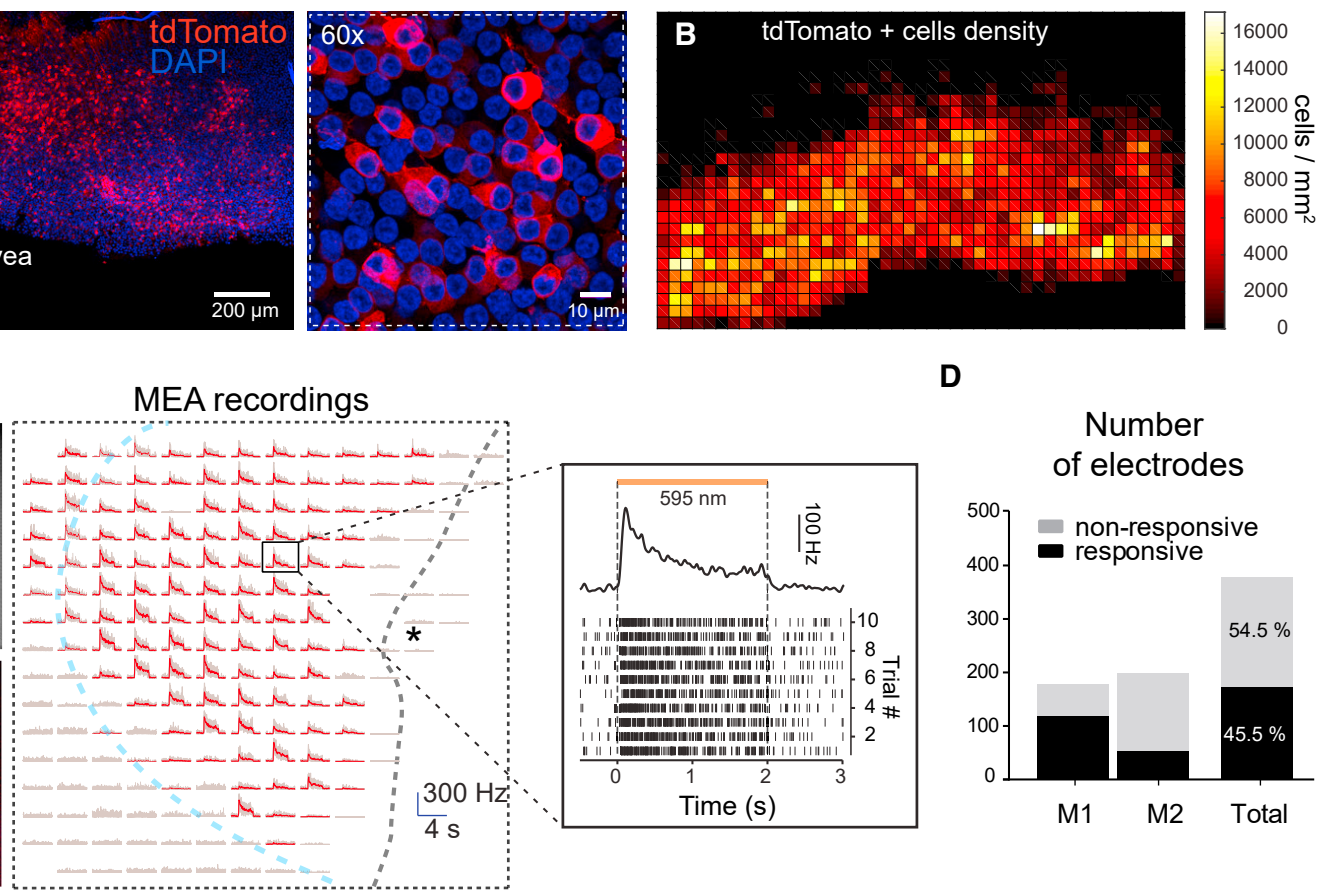

D
E

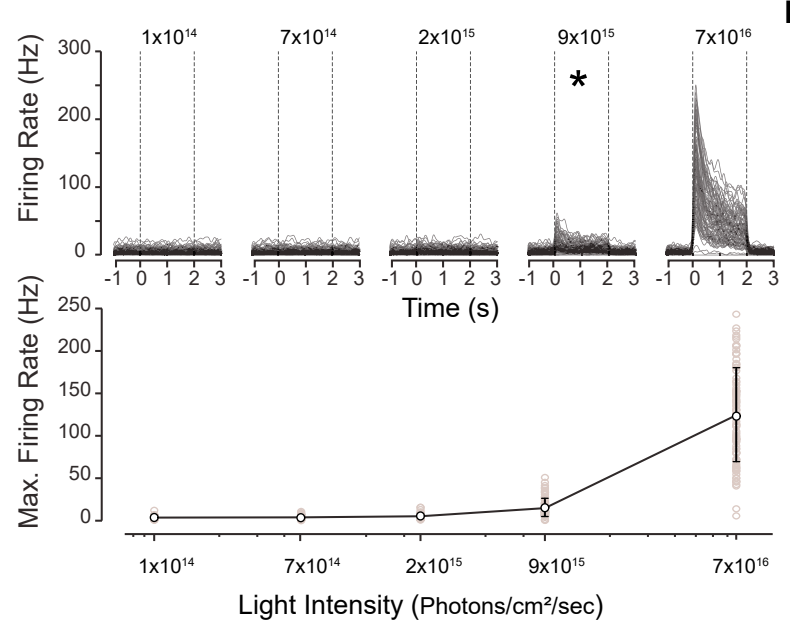

$\mathbf{F}$
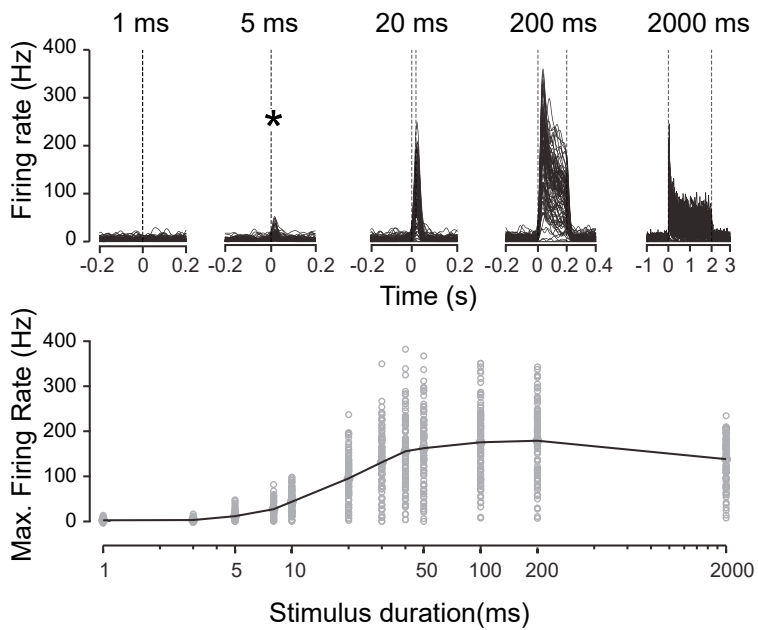

Figure 2. Expression and functionality: Multi-electrode array recordings

(A) Projection of confocal stack stitches (20x objective) showing the perifoveal area of M1 retina 20 months after treatment with a vector dose of $5 \times 10^{11}$ vg/eye (left). ChRtdT-expressing cells are shown in red, and the nuclei (in blue) are stained with DAPI. Scale bar: $200 \mu \mathrm{m}$. (Right) higher magnification image (60× objective). Scale bar: $10 \mu \mathrm{m}$. (B) Density map of ChR-tdT-positive RGCs for the hemifovea shown in (A). (C) Hemifovea RGC population recordings with an MEA (monkey M1). Top left, infrared view of the hemifovea with a 256-site MEA placed on the side of the RGC layer. Bottom left, epifluorescence image of the same retina showing tdTomato-ChrimsonR fluorescence. Right, global representation of the recorded RGC activity (firing rate) of the 256 sites during a 4-s period, with the application of a 2 -s flash of light (at a wavelength of $595 \mathrm{~nm}$ ). Right, inset, raster plot (bottom) and spike density function (top) of a representative recording site with 10 repeats. The dotted squares represent the MEA borders, the blue curves represent the approximate limits of ChrimsonR-tdTomato expression (half-donut shape), and the asterisks represent the center of the fovea. (D) Proportion of responsive versus non-responsive MEA electrodes for the retinae of the two primates (NHPs M1 and M2). (E) Firing rate (top) of 118 responsive electrodes (monkey M1 shown as an example) as a function of light stimulus intensity (black curves) and mean response intensity curve for these neurons (bottom). The asterisk represents the threshold light

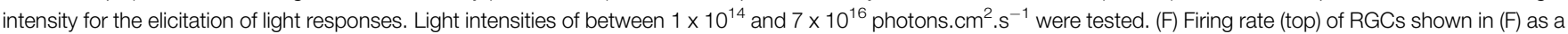
function of stimulus duration (black curves), and the mean population response curve (bottom). The asterisk represents the threshold light duration required to elicit light responses. Stimulus duration ranged from $1 \mathrm{~ms}$ to $2000 \mathrm{~ms}$. 

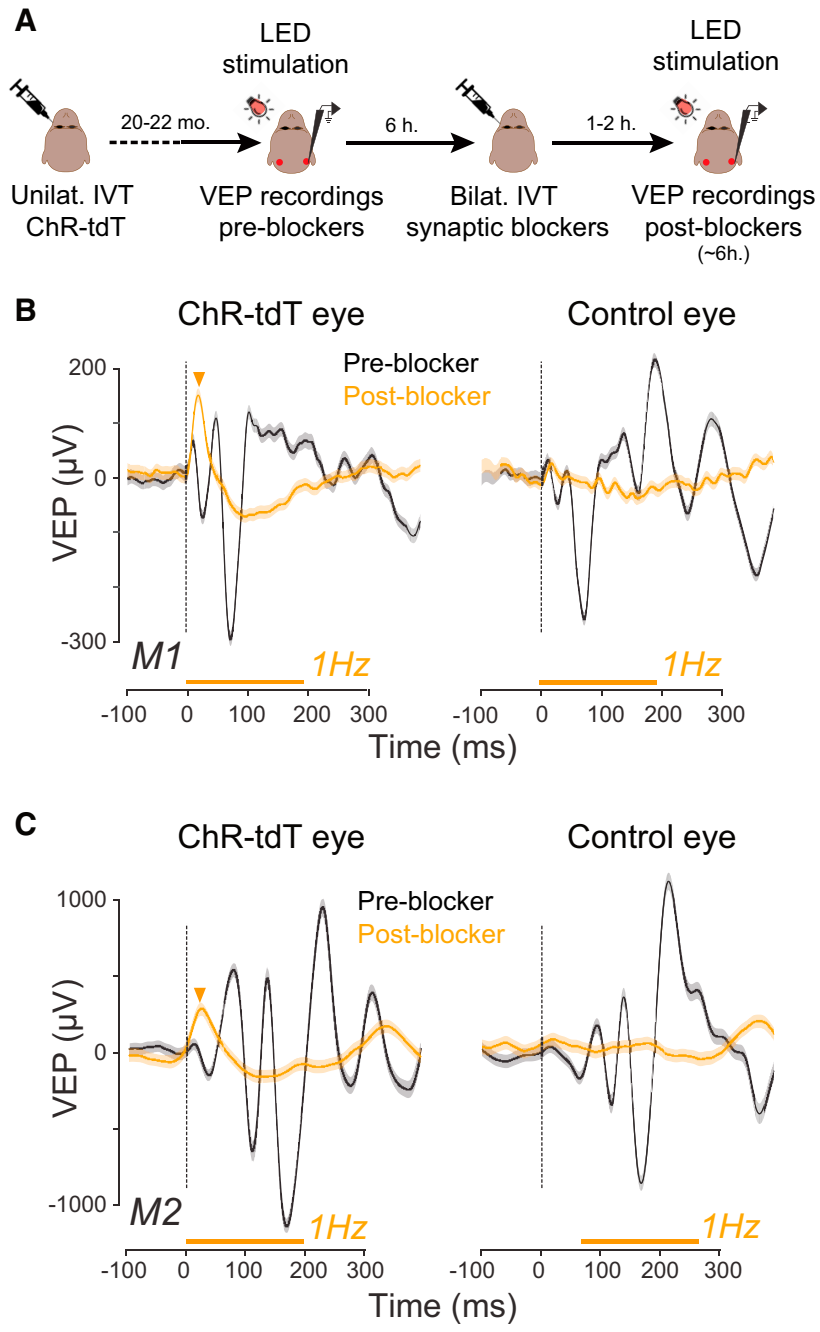

Figure 3. Cortical responses after retinal ChrimsonR optogenetic stimulation

(A) Experimental setup. Both animals received injections of AAV-ChrimsonRTdtomato into one eye. We recorded VEP responses 20-22 months after the injection, following various protocols of LED flash stimulation of the anesthetized animals during $\sim 6 \mathrm{~h}$. We then injected intravitrealy synaptic blockers (PDA + L-AP4) to block photoreceptor transmission. Finally, after a gap of $1-2 \mathrm{~h}$, we recorded VEP responses for the same LED flash stimulation protocols during $\sim 6 \mathrm{~h}$. (B) VEP responses to the orange LED before (black curves) and after (orange curves) the injection of synaptic blocker for monkey M1. The left panel shows VEP responses following stimulation of the ChR-tdT eye, and the right panel shows the response to stimulation of the control eye. Orange triangles indicate the peak latencies of VEP responses after treatment with a synapse blocker. (C) VEP responses for monkey M2 (same representation as in [B]). The dashed vertical lines indicate the onset of the light stimulation, and orange horizontal solid lines the duration of the stimulations.

With the synaptic block, light stimulation directly activates the engineered cells rather than the normal retinal pathway. In monkey M1, before the injection of the blocker, both VEP responses (black curves) were similar after stimulation of the ChR-tdT-expressing eye with an orange LED (595 nm, ChR-tdT eye, left panel) and for stimulation of the control eye (right panel), confirming that the sequence of orange flashes in both eyes activated the visual pathway naturally. After the intravitreal injection of synaptic blockers and stimulation of the eye with the same orange LED, we noted major changes in the shape of the recorded VEP. The VEP responses of the ChR-tdT-expressing eye (Figure $3 \mathrm{~B}$, orange traces) were characterized by an early positive phase (21 ms, orange arrow) and a late, sustained negative phase. Interestingly, stimulation of the control eye resulted in a stable recording, with no early positive or subsequent negative phase. Similar results were obtained with a second animal (monkey M2; Figure $3 \mathrm{C}$ ), with an early peak response at $24 \mathrm{~ms}$. The latency of the first positive peak was $21-24 \mathrm{~ms}$ for both animals, which is much shorter than the latencies classically reported for natural VEP components (positive components occur at 50-81 ms) and observed here. These data are consistent with the notion that these VEP responses in the visual cortex were elicited by the direct activation of ChR-tdT-expressing RGCs.

We then investigated the effects of several parameters, such as the light intensity and frequency of the orange LED, on VEP responses for both treated and control eyes (Figure 4). The amplitude of positive peak responses increased proportionally to light intensity (from 43 to $152 \mu \mathrm{V}$ for monkey M1 and from 121 to $221 \mu \mathrm{V}$ for monkey M2), and the peak latencies of these responses decreased with increasing light intensity (Figure 4B); from 82 to $24 \mathrm{~ms}$ for monkey M1 and 28 to $24 \mathrm{~ms}$ for monkey M2). No significant VEP response was recorded for the control eye (Figure 4C; post-blocker condition), even at maximal light intensity, confirming that the glutamate receptor antagonists used effectively abolished the natural light response. We then recorded VEP responses in response to an orange LED flashed at a frequency of $4 \mathrm{~Hz}$ (Figure 4D) and $16 \mathrm{~Hz}$ (Figure 4E) to confirm that activation occurred earlier after the optogenetic stimulation of RGCs. VEP peaks occurred more rapidly after blocker administration (orange traces) than in the absence of blocker (black traces), with similar time periods observed for stimulation at $4 \mathrm{~Hz}$ and at $1 \mathrm{~Hz}$ (30-37 ms for monkey M1; Figure 3B). For monkey M2, the difference in time to VEP peak was larger at $4 \mathrm{~Hz}(82-84 \mathrm{~ms})$ than at $1 \mathrm{~Hz}(\sim 58 \mathrm{~ms})$. After stimulation at $16 \mathrm{~Hz}$, VEP traces followed the train of pulses both before and after the injection of synaptic blocker. Although mean amplitudes of VEP responses were similar for stimulations at $1 \mathrm{~Hz}$ and $4 \mathrm{~Hz}$, they decreased drastically after stimulation at $16 \mathrm{~Hz}$ for both animals (Figure 4F). No such activation was observed following stimulation of the control eye, in either of the animals studied (Figures 4D and 4E; control eyes). These results demonstrate that optogenetic activation of the retina can trigger a transfer of information to higher visual centers, providing additional support for the potential of ChR-tdT for future therapeutic applications.

\section{DISCUSSION}

We previously showed that a single intravitreal injection of AAV2.7m8.CAG.ChrimsonR-tdT can efficiently target the foveal region of the retina, especially foveal midget RGCs, ${ }^{17}$ which are responsible for high-acuity vision. ${ }^{31}$ Using ex vivo live imaging and histology, we show here that expression persists in cells in the perifovea 
A

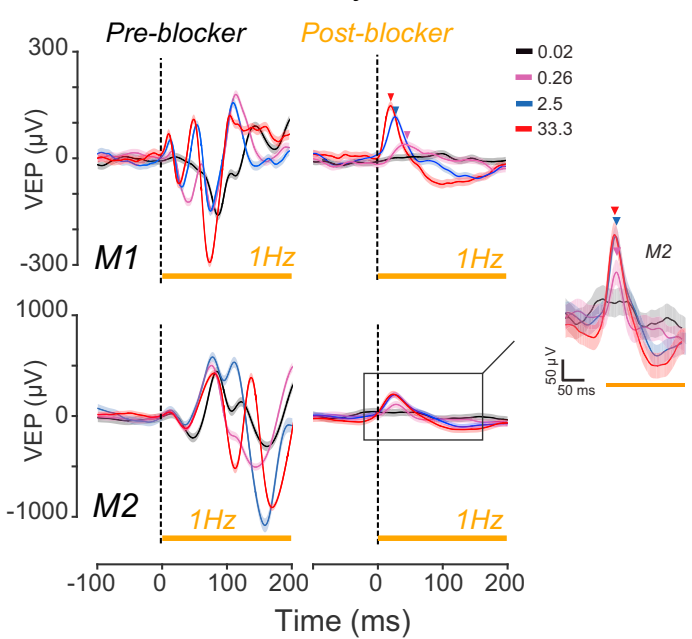

B

E

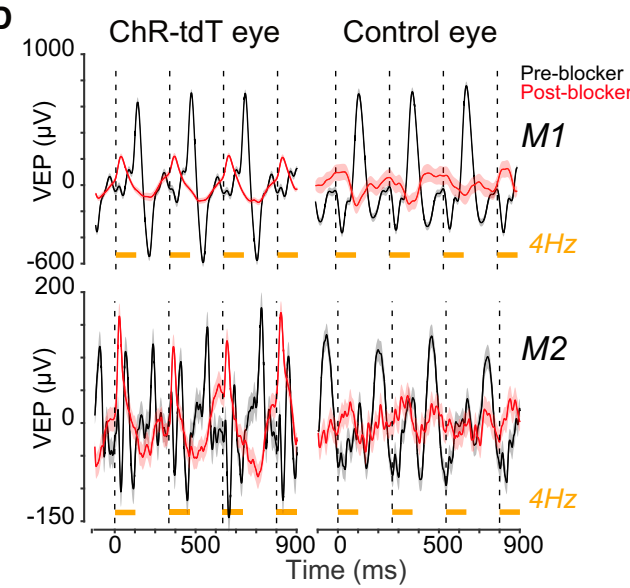

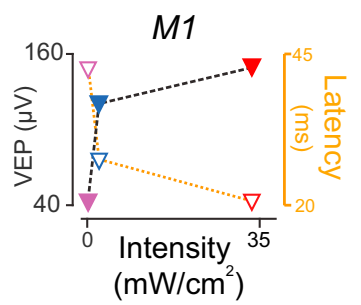

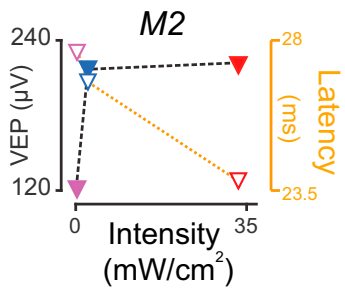

C
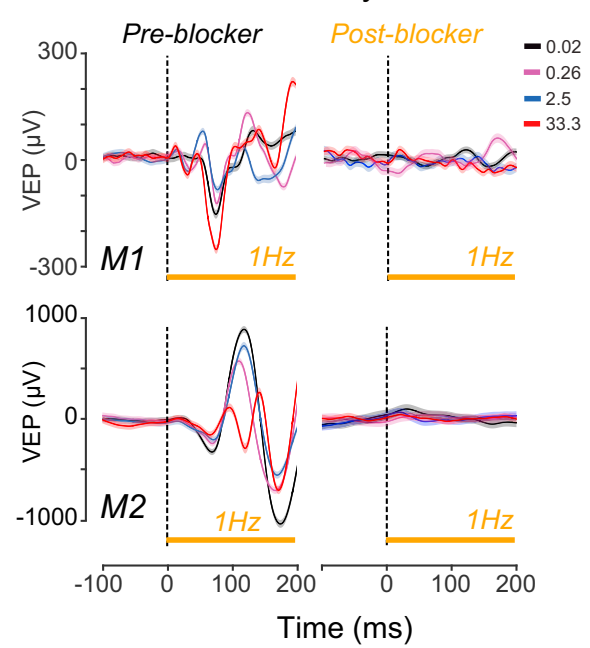

E

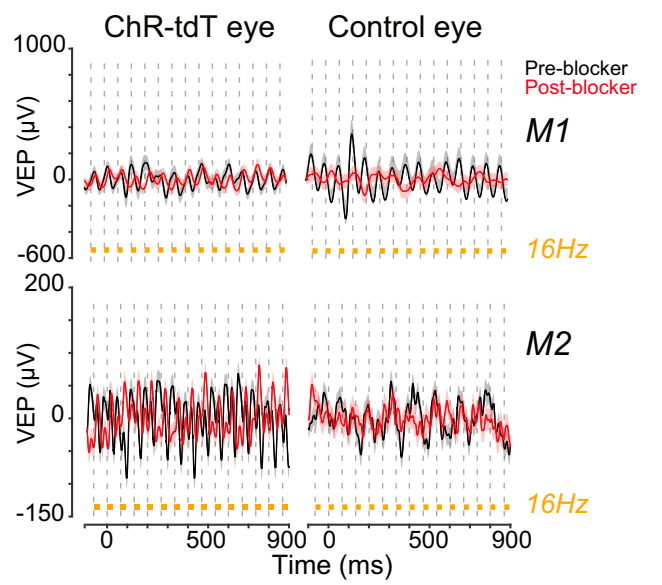

$\mathbf{F}$

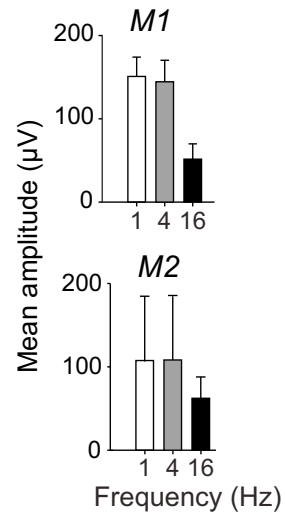

Figure 4. Cortical responses as a function of stimulus intensity and frequency

(A) VEP responses to stimulation of the ChR-tdT eye with different light intensities before and after injection of blockers. We tested four different intensities at a frequency of $1 \mathrm{~Hz}\left(33.3,2.5,0.26\right.$, and $\left.0.02 \mathrm{~mW} / \mathrm{cm}^{2}\right)$, as indicated by the color code. Top row, VEP responses for monkey M1. Bottom row, VEP responses for monkey M2. Each curve represents the mean VEP response and the SEM. The insert represents a magnification of monkey M2 post-blocker responses. Colored arrowheads indicate the maximum response amplitude detected at each light intensity in post-blocker condition. (B) Quantification of VEP responses peak amplitude and latency, as a function of light intensity in monkeys M1 (top) and M2 (bottom). Black dashed line with solid arrowheads represent response amplitudes, while orange dotted line with open arrowheads shows response latencies. (C) VEP responses in control eyes (same representation as in [A]). (D and E) VEP responses pre-blocker (black curves) and post-blocker (orange curves) for different light stimulation frequencies in treated or control eyes for both animals. Two different light frequencies (4 Hz in [D] and $16 \mathrm{~Hz}$ in [E]) were tested with a light intensity of $33.3 \mathrm{~mW} /$ $\mathrm{cm}^{2}$. (F). Mean amplitudes of positive phases of VEP responses for different light frequencies (1, 4, and $16 \mathrm{~Hz}$ ) for monkey 1 (top, M1) and monkey 2 (bottom, M2). Errors bars indicate SEM. The dashed vertical lines indicate the onset of the light stimulation, and orange solid horizontal lines the duration of the stimulations (200 ms for $1 \mathrm{~Hz}, 125 \mathrm{for}$ $4 \mathrm{~Hz}$, and $32 \mathrm{~ms}$ for $16 \mathrm{~Hz})$.

region more than 20 months after the injection, and we demonstrate, with electrophysiological recordings, that these transfected RGCs remain functional, displaying rapid, robust responses. Previous studies based on ex vivo retinal recordings in NHP models have demonstrated efficient optogene expression for up to 6 months, ${ }^{9,15,17}$ and a study based on retinal imaging in vivo extended this functional window up to 14 months. ${ }^{20}$ This maintenance of activity so long after the injection is consistent with the notion that gene therapy can lead to long-term gene expression. Furthermore, despite the absence of immunohistopathological results in our dataset, it provides additional evidence that the microbial opsin ChR-tdT does not induce an immune response that might eventually destroy the engineered RGCs. Indeed, the RGC responses were in a range similar to that recorded in our initial experiments, ${ }^{17}$ and transfected cell density was also at similar levels. However, given the small number of replicates in this study and the considerable inter-subject variability previously observed after 2 or 6 months, it is difficult to make quantitative comparisons with earlier expression time points. 
We demonstrate here, through in vivo VEP recordings, that the selective stimulation of transfected RGCs induces specific cortical responses. Based on our retinal observations, we can interpret these VEP recordings as reflecting the activation of cortical neurons due to the direct functional optogenetic activation of RGCs, leading to the synaptic transfer of information to cortical neurons. All previous studies on optogenetic RGC activation in the primate retina were performed on RGCs either ex vitro ${ }^{9,15,17}$ or in vivo. ${ }^{20}$ The recorded rates of RGC firing activity and the reported increases in calcium indicator fluorescence were highly suggestive of potential information transfer to the higher visual centers, but no experimental demonstration for the existence of this communication was provided. While our pharmacological blockers treatment does not recapitulate the extent of retinal degeneration diseases (i.e., cell loss and aberrant neuronal connectivity), it does cancel VEP recorded in control eyes and reveals the specific VEP responses elicited by direct optogenetic activation of RGCs in treated eyes. Here, by blocking the glutamatergic synaptic transmission in the retina, the optogenetic VEP responses had an earlier peak response than in normal VEPs. Such early cortical responses were recorded in blind rodents during optogenetic activation of either the dormant cones, ${ }^{14}$ the bipolar cells, ${ }^{18,19}$ or the RGCs. ${ }^{15,27}$ This short VEP latency, recorded in absence of the photoreceptors signal transmission, does not fit with intrinsically photosensitive RGC (ipRGC) kinetic characteristics. Indeed, melanopsin-driven RGC light responses are consistently slower. ${ }^{34}$ Although we cannot exclude a potential contribution of ipRGCs in our recordings, since blockers should not have a significant impact on these cells, ${ }^{35}$ the short VEP response latencies observed are most likely the signature of direct optogenetic stimulation within the inner retina. The optogenetic activation would occur more rapidly than natural responses, due to the relative slowness of the phototransduction cascade and of synaptic information transfer between the different retinal layers. We showed that cortical VEP responses increased with light intensity. This result is highly consistent with the RGC spike recording on the isolated retina, with a clear relationship between RGC activity and light intensity. Given the high light levels used in our VEP experiments, the observed increases in VEP peak amplitudes with increasing light intensity are consistent with an optogenetic origin, because any residual natural light responses would be fully saturated at such light intensities. Finally, these VEP recordings show that optogenetic responses can follow frequencies of at least $16 \mathrm{~Hz}$, as expected from the high temporal resolution achieved with RGCs in ex vivo single-cell recordings (Figure $1 \mathrm{H}$ ). All these VEP recordings validate the therapeutic potential of ChR-tdT for restoring vision in blind patients.

If the recorded signal in the cortex is most probably of optogenetic origin, it is hard to estimate the degree of information it carries. The waveform of the signal is very different from the control recording and shows a simpler dynamic and lower amplitude. The simple biphasic response obtained after blockade might be explained by the functional bypass of outer retina circuitry that converts all the RGCs expressing the optogene into ON cells, independently of their actual type. Importantly, this also occurs with retinal electrical im- plants restoration strategies that produce RGC excitations. ${ }^{1}$ While deeply changing the activity output of the retina, these implants have been shown to elicit visual responses, nonetheless. ${ }^{2}$ Concerning the variation in VEP response amplitudes, only a portion of the fovea RGCs are expressing ChrimsonR; as such, only a fraction of total retinal cells contribute to the VEPs isolated under synaptic blockade compared to pre-blocker conditions. In this regard, the decrease in signal amplitude to light flashes under blockers in ChR-tdT eyes compared to pre-blocker recordings is not surprising.

In conclusion, this study describes the cortical effect of an optogenetic stimulation of the primate retina and raises hopes of long-term functionality for optogenetic approaches in blind patients, who may benefit from this therapy. ${ }^{36}$ It also opens up new avenues of research into the neural integration and computations occurring at the cortical level in NHPs, with a view to restoring the sensitivity of sensory organs through optogenetics.

\section{MATERIALS AND METHODS \\ Animals}

Data were collected for three captive-born macaques (Macaca fascicularis; two males, monkey M1, monkey M2, weighing 3.2 , and $3.9 \mathrm{~kg}$, respectively; one female, monkey $\mathrm{M} 0,4.1 \mathrm{~kg}$ ). Monkeys were housed in pairs and handled in strict accordance with the recommendations of the Weatherall Report on good animal practice. Monkey housing conditions, surgical procedures and experimental protocols were performed in strict accordance with the National Institutes of Health Guidelines (1996) and after validation of the European Council Directive (2010/63/EU), and the study was approved by the French government and institutional and regional committees for animal care (Committee C. Darwin, registration \#9013). Our routine laboratory procedures included an environmental enrichment program, in which the monkeys were allowed visual, auditory, and olfactory contact with other animals and, when appropriate, could touch and groom each other.

\section{AAV production}

ChrimsonR-tdTomato was inserted into an AAV backbone plasmid. The construct included a woodchuck hepatitis virus posttranscriptional regulatory element and bovine growth hormone poly(A) sequences. Recombinant AAVs were produced by the plasmid cotransfection method, ${ }^{34}$ and the resulting lysates were purified by iodixanol gradient ultracentrifugation, as previously described. Briefly, the $40 \%$ iodixanol fraction was concentrated and subjected to buffer exchange with Amicon Ultra-15 Centrifugal Filter Units. Vector stocks were then titered for DNase-resistant vector genomes by real-time PCR relative to a standard. ${ }^{37}$

\section{Gene delivery}

Primates were anesthetized with 10:1 mg/kg mixture of ketamine and xylazine. We injected $100 \mu \mathrm{L}$ of viral vector suspension into the vitreous of one eye in each animal. Following the injection, an ophthalmic steroid and an antibiotic ointment were applied to the cornea. Experiments were conducted 21,20 , and 22 months after injection for M0, M1, and 
M2, respectively. None of the treated animals displayed any sign of photophobia or vision-related behavioral changes during housing.

\section{Recording of VEPs}

We performed in vivo VEP recordings 20 to 22 months after AAV injection, during a terminal experiment in two animals (M1 and M2). Briefly, anesthesia was induced with ketamine $(0.2 \mathrm{mg} / \mathrm{kg}$, intramuscular [i.m.]) and dexmedetomidine (0.015 mg/kg, i.m.) and maintained with alfaxan $(0.1 \mathrm{mg} / \mathrm{kg} /, \mathrm{min}$, i.v. $)$. The monkey was placed in a stereotaxic frame, and heart rate, temperature, respiration and peripheral oxygen saturation were monitored throughout the experiment. Eye drops were used to dilate the pupils ( $0.5 \%$ tropicamide). Lubrithal eye gel was used to maintain good corneal moisture. We placed four electrodes in subcutaneous positions: two at each temple and two at each occipital operculum (left and right), and we set the high- and low-path filters to $50 \mathrm{~Hz}$ and $0.05 \mathrm{~Hz}$, respectively, on an RZ-2 amplifier (Tucker Davis, Alachua, FL, USA). Light stimuli were generated with an orange LED (M595L3 from Thorlab, $595 \mathrm{~nm}$ ) equipped with a plano-convex lens for collimation (LA1805-A-ML, Thorlabs). The LED was positioned $3 \mathrm{~cm}$ away from the eye, and the light spot was centered on the dilated pupil. $\mathrm{VEP}$ responses were recorded before and after the intravitreal injection of synaptic blockers-PDA and L-AP4-used to block natural light responses. ${ }^{38}$ We injected intravitreally $100 \mu \mathrm{L}$ of a PBS solution containing PDA $(200 \mathrm{mM})$ and L-AP4 $(40 \mathrm{mM})$. The fascicularis eyeball has a $2.1-\mathrm{mL}$ volume of aqueous humor, ${ }^{39}$ leading, at full dilution, to a final concentration of $10 \mathrm{mM}$ PDA and $2 \mathrm{mM}$ LAP4. The two eyes were injected at the same time (inside a 7-min window), and we started recording VEPs in response to light flashes at 1-2 $\mathrm{h}$ post injection and finished acquisition at +7 to $+8 \mathrm{~h}$. For both animals, we started recordings responses on the control eye and then on the injected eye $(+1$ or +2 to $\sim 5 \mathrm{~h}$ control eye, $\sim 5$ to +7 or $+8 \mathrm{~h}$ treated eye). The eyes of the monkeys were subjected to flash stimuli at various intensities (from 0.02 to $33.3 \mathrm{~mW} / \mathrm{cm}^{2}$ ) and frequencies (1, 4 , and $16 \mathrm{~Hz}$ ). The duration of the stimuli was 200,125 , and $32 \mathrm{~ms}$ for frequencies of 1,4 , and $16 \mathrm{~Hz}$, respectively. We made 300 consecutive recordings of each VEP response and then averaged response waveforms for each VEP measurement. VEP responses were similar for the ipsilateral and contralateral electrodes. We therefore present VEP responses for the electrode contralateral to the injected eye only.

\section{Primate retina isolation and preservation}

After the in vivo VEP recordings, the primates received a lethal dose of pentobarbital. Their eyeballs were removed, perforated with a sterile 20-gauge needle and placed in sealed bags with $\mathrm{CO}_{2}$ independent medium (Thermo Fisher scientific) for transport. The retinae were isolated, and the RPE was removed and stored as retinal explants in an incubator for $\sim 24 \mathrm{~h}$ before recording. Hemifoveal retina fragments were transferred to Neurobasal + B27 medium in polycarbonate Transwell microporous membrane inserts (Corning) for conservation in the cell culture incubator. These hemifoveal regions were subsequently used for simultaneous single-cell and MEA recordings. We abolished all natural responses entirely, by applying pharmacological blockers (see below).

\section{Two-photon live imaging and single-cell electrophysiological recordings}

A custom-built two-photon microscope equipped with a $25 \times$ water immersion objective (XLPLN25xWMP, NA: 1.05, Olympus) with a pulsed femtosecond laser (InSight DeepSee, Newport Corporation) was used for imaging ChR-tdT-positive RGCs. AAV-treated macaque retinas were imaged in oxygenized $\left(95 \% \mathrm{O}_{2}, 5 \% \mathrm{CO}_{2}\right)$ Ames medium (Sigma-Aldrich). For live two-photon imaging, whole-mount retinas (without the RPE attached) were placed in the recording chamber of the microscope (ganglion cell layer side up), and images and $z$ stacks were acquired with the excitation laser at a wavelength of $1,050 \mathrm{~nm}$. Images were processed offline with ImageJ.

We used an Axon Multiclamp 700B amplifier for whole-cell patchclamp and cell-attached recordings. Patch electrodes were made from borosilicate glass (BF100-50-10, Sutter Instruments) and pulled to 7$9 \mathrm{M} \Omega$. Pipettes were filled with $115 \mathrm{mM}$ potassium gluconate, $10 \mathrm{mM}$ $\mathrm{KCl}, 1 \mathrm{mM} \mathrm{MgCl} 2,0.5 \mathrm{mM} \mathrm{CaCl}_{2}, 1.5 \mathrm{mM}$ EGTA, $10 \mathrm{mM}$ HEPES, and $4 \mathrm{mM}$ ATP- $\mathrm{Na}_{2}$ (pH 7.2). We clamped the cells at a potential of $-60 \mathrm{mV}$, to isolate excitatory currents. Recordings were also performed in the loose-patch configuration with the pipettes filled with Ames medium, to record spiking activity. The retinae were dark-adapted for at least $30 \mathrm{~min}$ in the recording chamber before recordings. AMPA/kainate glutamate receptor antagonist, 6-cyano-7-nitroquinoxaline-2,3dione (CNQX, $25 \mu \mathrm{M}$, Sigma-Aldrich), NMDA glutamate receptor antagonist, [3H]3-(2-carboxypiperazin-4-yl) propyl-1-phosphonic acid (CPP, $10 \mu \mathrm{M}$, Sigma- Aldrich), and a selective group III metabotropic glutamate receptor agonist, L-(+)-2-amino-4-phosphonobutyric acid (L-AP4, $50 \mu \mathrm{M}$, Tocris Bioscience, Bristol, UK) were diluted to the appropriate concentration from stock solutions and added to the Ames medium before recordings.

\section{MEA}

MEA recordings were obtained for retinal fragments (without the RPE attached) placed on a cellulose membrane that had been incubated with polylysine $(0.1 \%$, Sigma) overnight. Once on the micromanipulator, the piece of retina was gently pressed against an MEA (MEA256 100/30 iR-ITO; Multi-Channel Systems, Reutlingen, Germany), with the RGCs toward the electrodes. We measured tdTomato fluorescence to check that the retina was correctly positioned before making recordings under a Nikon Eclipse Ti inverted microscope (Nikon, Dusseldorf, Germany) with the MEA system mounted on the stage. The retina was continuously perfused with Ames medium (Sigma-Aldrich, St Louis, MO) bubbled with $95 \%$ $\mathrm{O}_{2}$ and $5 \% \mathrm{CO}_{2}$ at $34^{\circ} \mathrm{C}$, at a rate of $1-2 \mathrm{~mL} / \mathrm{min}$ during experiments. AMPA/kainate glutamate receptor antagonist, 6-cyano-7-nitroquinoxaline-2,3-dione (CNQX, $25 \mu \mathrm{M}$, Sigma-Aldrich), NMDA glutamate receptor antagonist, [3H]3-(2-carboxypiperazin-4-yl) propyl-1-phosphonic acid (CPP, $10 \mu \mathrm{M}$, Sigma- Aldrich), and a selective group III metabotropic glutamate receptor agonist, L-(+)-2amino-4-phosphonobutyric acid (L-AP4, $50 \mu \mathrm{M}$, Tocris Bioscience, Bristol, UK) were diluted to the appropriate concentration from stock solutions and added to the bath through the perfusion system for $10 \mathrm{~min}$ before recordings. Action potentials were identified on 
the filtered electrode signal (second-order high-pass Butterworth, cutoff frequency $200 \mathrm{~Hz}$ ), with a threshold of 4 times the SD of the signal. Spike density function was calculated, averaged over repeat stimulations, and used to determine the maximal firing rate over a time window corresponding to the duration of the stimulus plus $50 \mathrm{~ms}$. In comparisons of responses between light intensities, we calculated, for each electrode, the added firing rate as the maximal firing rate minus the spontaneous firing rate for the electrode concerned, which was calculated as the mean firing rate in the $2 \mathrm{~s}$ before stimulation.

\section{Photostimulation for ex vivo experiments}

For single-cell electrophysiological recordings, full-field photostimulation was performed with a Polychrome $\mathrm{V}$ monochromator (Olympus, Hamburg, Germany) set to $595 \mathrm{~nm}( \pm 10 \mathrm{~nm})$, and output light intensities were calibrated and ranged from $5.8 \times 10^{14}$ to $3.15 \mathrm{x}$ $10^{17}$ photons. $\mathrm{cm}^{2} . \mathrm{s}^{-1}$. For spectral sensitivity experiments, stimulation wavelengths between 400 and $650 \mathrm{~nm}$ were tested, in $25-\mathrm{nm}$ steps. For flicker stimulation, 10 repeats were used in full duty cycle, at frequencies ranging from $2 \mathrm{~Hz}$ to $28 \mathrm{~Hz}$. For MEA recordings, fullfield light stimuli were applied with another Polychrome V monochromator set to $595 \mathrm{~nm}( \pm 10 \mathrm{~nm})$, driven by a STG2008 stimulus generator (MCS). Output light intensities were calibrated and ranged from $1.37 \times 10^{14}$ to $6.78 \times 10^{16}$ photons. $\mathrm{cm}^{2} . \mathrm{s}^{-1}$. For intensity curves, we used 2-s flashes at five intensities $\left(1.37 \times 10^{14}, 6.56 \times 10^{14}, 2.34 \mathrm{x}\right.$ $10^{15}, 8.82 \times 10^{15}, 6.78 \times 10^{16}$ photons. $\left.\mathrm{cm}^{2} . \mathrm{s}^{-1}\right)$, each repeated 10 times, with a 5 -s interval between stimuli. For the duration of stimulation assay, we used 12 different durations (ranging from 1 to $2000 \mathrm{~ms}$ ), each repeated 10 times, with a 5-s interval between stimuli. Calibrations were performed with a spectrophotometer (USB2000+, Ocean Optics, Dunedin, FL).

\section{Confocal imaging and quantification}

After MEA experiments, the tissue was recovered and fixed by incubation with $4 \%$ paraformaldehyde for $30 \mathrm{~min}$ at room temperature, rinsed with $\mathrm{PBS}$, and stored at $4^{\circ} \mathrm{C}$ in sodium azide solution. Hemifoveas were then mounted in Vectashield containing DAPI (H-1000, Vector Laboratories) on slides and covered with a coverslip $(18 \times 18 \mathrm{~mm}$, Biosigma), using a $100-\mu \mathrm{m}$ spacer (Secure-seal space S24735, Thermo Fisher Scientific), which was sealed with nail polish. The retinas were imaged on an inverted confocal microscope (Fluoview 1200 , Olympus), with a $20 \times$ objective (UPLSAPO 20XO, NA: 0.85 , Olympus), voxel sizes of $0.265-0.388 \mu \mathrm{m} /$ pixel in the $x$ and $y \mathrm{di}$ rections and $1.64 \mu \mathrm{m} /$ pixel in the $z$ direction. For each hemifovea, we recorded multiple stacks and reconstituted an automatic stitch (10\% overlap). Using Td-tomato fluorescence, we performed manual 3D counts of the transfected cells in ImageJ (http://imagej.nih.gov/ij) with the cell counter plugin. The results were then processed with custom-developed MATLAB analysis software for the calculation of local density.

\section{SUPPLEMENTAL INFORMATION}

Supplemental information can be found online at https://doi.org/10. 1016/j.omtm.2021.11.009.

\section{ACKNOWLEDGMENTS}

We thank Morgane Weissenburger, Estelle Chavret-Reculon, Lucile Aubree, Corina Dussaud, and Benedicte Daboval for their superb animal care. We thank Valérie Fradot for technical assistance with primate tissue preparation. This project was supported by Bpifrance (grant reference 2014-PRSP-15), Gensight Biologics, Foundation Fighting Blindness, Fédération des Aveugles de France, and by French state funds managed by the Agence Nationale de la Recherche within the Investissements d'Avenir program, RHU LIGHT4DEAF [ANR15-RHU-0001], LABEX LIFESENSES [ANR- 10-LABX-65], IHU FOReSIGHT [ANR-18-IAHU-0001], [ANR-11-IDEX-0004-02].

\section{AUTHOR CONTRIBUTIONS}

Conceptualization, A.C., S.P., F.A.and G.G.; methodology and performance of experiments, A.C., M.P., C.J., K.B., G.L., R.G., E.Bu., E.Br., F.A., and G.G.; formal analysis, A.C., C.J., G.G., and F.A.; writing original draft, A.C., F.A., G.G., and S.P.; writing - review \& editing, A.C., F.A., G.G., P.P., and S.P.; supervision and funding, J-A.S., S.P., F.A., and G.G.

\section{DECLARATION OF INTERESTS}

S.P. is a consultant for Gensight Biologics; J-A.S. and S.P. have financial interests in Gensight Biologics. G.G., J-A.S., and S.P. have filed a patent application relating to the gene therapy construct presented here.

\section{REFERENCES}

1. Palanker, D., Le Mer, Y., Mohand-Said, S., Muqit, M., and Sahel, J.A. (2020) Photovoltaic restoration of central vision in atrophic age-related macular degeneration. Ophthalmology 127, 1097-1104.

2. Prévot, P.H., Gehere, K., Arcizet, F., Akolkar, H., Khoei, M.A., Blaize, K., Oubari, O. Daye, P., Lanoë, M., Valet, M., et al. (2020). Behavioural responses to a photovoltaic subretinal prosthesis implanted in non-human primates. Nat. Biomed. Eng. 4, $172-180$.

3. Stingl, K., Schippert, R., Bartz-Schmidt, K.U., Besch, D., Cottriall, C.L., Edwards, T.L. Gekeler, F., Greppmaier, U., Kiel, K., Koitschev, A., et al. (2017). Interim results of a multicenter trial with the new electronic subretinal implant alpha AMS in 15 patients blind from inherited retinal degenerations. Front. Neurosci. 11, 445.

4. da Cruz, L., Dorn, J.D., Humayun, M.S., Dagnelie, G., Handa, J., Barale, P.O., Sahel, J.A., Stanga, P.E., Hafezi, F., Safran, A.B., et al. (2016). Five-year safety and performance results from the argus II retinal prosthesis system clinical trial. Ophthalmology 123, 2248-2254.

5. Shirai, H., Mandai, M., Matsushita, K., Kuwahara, A., Yonemura, S., Nakano, T. Assawachananont, J., Kimura, T., Saito, K., Terasaki, H., et al. (2016). Transplantation of human embryonic stem cell-derived retinal tissue in two primate models of retinal degeneration. Proc. Natl. Acad. Sci. U S A 113, E81-E90.

6. Barnea-Cramer, A.O., Wang, W., Lu, S.J., Singh, M.S., Luo, C., Huo, H., McClements, M.E., Barnard, A.R., MacLaren, R.E., and Lanza, R. (2016). Function of human pluripotent stem cell-derived photoreceptor progenitors in blind mice. Sci. Rep. 6, $1-15$

7. Garita-Hernandez, M., Lampič, M., Chaffiol, A., Guibbal, L., Routet, F., SantosFerreira, T., Gasparini, S., Borsch, O., Gagliardi, G., Reichman, S., et al. (2019). Restoration of visual function by transplantation of optogenetically engineered photoreceptors. Nat. Commun. 10, 1-13.

8. Gagliardi, G., Ben M'Barek, K., and Goureau, O. (2019). Photoreceptor cell replacement in macular degeneration and retinitis pigmentosa: a pluripotent stem cell-based approach. Prog. Retin. Eye Res. 71, 1-25. 
9. Ivanova, E., Hwang, G.S., Pan, Z.H., and Troilo, D. (2010). Evaluation of AAV-mediated expression of chop2-GFP in the marmoset retina. Invest. Ophthalmol. Vis. Sci. $51,5288-5296$.

10. Bi, A., Cui, J., Ma, Y.P., Olshevskaya, E., Pu, M., Dizhoor, A.M., and Pan, Z.H. (2006) Ectopic expression of a microbial-type rhodopsin restores visual responses in mice with photoreceptor degeneration. Neuron 50, 23-33.

11. Gaub, B.M., Berry, M.H., Holt, A.E., Isacoff, E.Y., and Flannery, J.G. (2015). Optogenetic vision restoration using rhodopsin for enhanced sensitivity. Mol. Ther. 23, 1562-1571.

12. Cehajic-Kapetanovic, J., Eleftheriou, C., Allen, A.E., Milosavljevic, N., Pienaar, A., Bedford, R., Davis, K.E., Bishop, P.N., and Lucas, R.J. (2015). Restoration of vision with ectopic expression of human rod opsin. Curr. Biol. 25, 2111-2122.

13. Berry, M.H., Holt, A., Salari, A., Veit, J., Visel, M., Levitz, J., Aghi, K., Gaub, B.M., Sivyer, B., Flannery, J.G., et al. (2019). Restoration of high-sensitivity and adapting vision with a cone opsin. Nat. Commun. 10, 1-12.

14. Busskamp, V., Duebel, J., Balya, D., Fradot, M., Viney, T.J., Siegert, S., Groner, A.C., Cabuy, E., Forster, V., Seeliger, M., et al. (2010). Genetic reactivation of cone photoreceptors restores visual responses in retinitis pigmentosa. Science 329, 413-417.

15. Chaffiol, A., Caplette, R., Jaillard, C., Brazhnikova, E., Desrosiers, M., Dubus, E., Duhamel, L., Macé, E., Marre, O., Benoit, P., et al. (2017). A new promoter allows optogenetic vision restoration with enhanced sensitivity in macaque retina. Mol. Ther. $25,2546-2560$.

16. Gaub, B.M., Berry, M.H., Holt, A.E., Reiner, A., Kienzler, M.A., Dolgova, N., Nikonov, S., Aguirre, G.D., Beltran, W.A., Flannery, J.G., et al. (2014). Restoration of visual function by expression of a light-gated mammalian ion channel in retinal ganglion cells or ON-bipolar cells. Proc. Natl. Acad. Sci. U S A 111, E5574-E5583.

17. Gauvain, G., Akolkar, H., Chaffiol, A., Arcizet, F., Khoei, M.A., Desrosiers, M. Jaillard, C., Caplette, R., Marre, O., Bertin, S., et al. (2021). Optogenetic therapy: high spatiotemporal resolution and pattern discrimination compatible with vision restoration in non-human primates. Commun. Biol. 4, 1-15.

18. Lagali, P.S., Balya, D., Awatramani, G.B., Münch, T.A., Kim, D.S., Busskamp, V., Cepko, C.L., and Roska, B. (2008). Light-activated channels targeted to ON bipolar cells restore visual function in retinal degeneration. Nat. Neurosci. 11, 667-675.

19. Macé, E., Caplette, R., Marre, O., Sengupta, A., Chaffiol, A., Barbe, P., Desrosiers, M. Bamberg, E., Sahel, J.A., Picaud, S., et al. (2015). Targeting channelrhodopsin-2 to ON-bipolar cells with vitreally administered AAV restores on and off visual responses in blind mice. Mol. Ther. 23, 7-16.

20. McGregor, J.E., Godat, T., Dhakal, K.R., Parkins, K., Strazzeri, J.M., Bateman, B.A., Fischer, W.S., Williams, D.R., and Merigan, W.H. (2020). Optogenetic restoration of retinal ganglion cell activity in the living primate. Nat. Commun. 11, 1-9.

21. Lin, B., Koizumi, A., Tanaka, N., Panda, S., and Masland, R.H. (2008). Restoration of visual function in retinal degeneration mice by ectopic expression of melanopsin. Proc. Natl. Acad. Sci. U S A 105, 16009-16014.

22. Wright, A.F., Chakarova, C.F., Abd El-Aziz, M.M., and Bhattacharya, S.S. (2010). Photoreceptor degeneration: genetic and mechanistic dissection of a complex trait. Nat. Rev. Genet. 11, 273-284.

23. Khabou, H., Garita-Hernandez, M., Chaffiol, A., Reichman, S., Jaillard, C., Brazhnikova, E., Bertin, S., Forster, V., Desrosiers, M., Winckler, C., et al. (2018). Noninvasive gene delivery to foveal cones for vision restoration. JCI Insight 3, e96029.
24. Jacobson, S.G., Sumaroka, A., Luo, X., and Cideciyan, A.V. (2013). Retinal optogenetic therapies: clinical criteria for candidacy. Clin. Genet. 84, 175-182.

25. Picaud, S., Dalkara, D., Marazova, K., Goureau, O., Roska, B., and Sahel, J.A. (2019). The primate model for understanding and restoring vision. Proc. Natl. Acad. Sci. U S A $116,26280-26287$.

26. Isago, H., Sugano, E., Wang, Z., Murayama, N., Koyanagi, E., Tamai, M., and Tomita, H. (2012). Age-dependent differences in recovered visual responses in Royal College of Surgeons rats transduced with the Channelrhodopsin-2 gene. J. Mol. Neurosci. 46, 393-400.

27. Sato, M., Sugano, E., Tabata, K., Sannohe, K., Watanabe, Y., Ozaki, T., Tamai, M., and Tomita, H. (2017). Visual responses of photoreceptor-degenerated rats expressing two different types of channelrhodopsin genes. Sci. Rep. 7, 1-10

28. Arens-Arad, T., Farah, N., Lender, R., Moshkovitz, A., Flores, T., Palanker, D., and Mandel, Y. (2020). Cortical interactions between prosthetic and natural vision. Curr. Biol. 30, 176-182.e2

29. Le Meur, G., Lebranchu, P., Billaud, F., Adjali, O., Schmitt, S., Bézieau, S., Péréon, Y. Valabregue, R., Ivan, C., Darmon, C., et al. (2018). Safety and long-term efficacy of AAV4 gene therapy in patients with RPE65 leber congenital amaurosis. Mol. Ther. $26,256-268$.

30. Gauvain, G., and Murphy, G.J. (2015). Projection-specific characteristics of retinal input to the brain. J. Neurosci. 35, 6575-6583.

31. Dacey, D.M., and Petersen, M.R. (1992). Dendritic field size and morphology of midget and parasol ganglion cells of the human retina. Proc. Natl. Acad. Sci. U S A $89,9666-9670$.

32. Klapoetke, N.C., Murata, Y., Kim, S.S., Pulver, S.R., Birdsey-Benson, A., Cho, Y.K. Morimoto, T.K., Chuong, A.S., Carpenter, E.J., Tian, Z., et al. (2014). Independent optical excitation of distinct neural populations. Nat. Methods 11, 338-346.

33. Hecht, S., and Verrijp, C.D. (1933). The influence of intensity, color and retinal location on the fusion frequency of intermittent illumination. Proc. Natl. Acad. Sci. U S A $19,522-535$.

34. Mure, L.S. (2021). Intrinsically photosensitive retinal ganglion cells of the human retina. Front. Neurol. 12, 1-10

35. Gamlin, P.D.R., McDougal, D.H., Pokorny, J., Smith, V.C., Yau, K.W., and Dacey, D.M. (2007). Human and macaque pupil responses driven by melanopsin-containing retinal ganglion cells. Vis. Res. 47, 946-954.

36. Sahel, J.A., Boulanger-Scemama, E., Pagot, C., Arleo, A., Galluppi, F., Martel, J.N. Esposti, S.D., Delaux, A., de Saint Aubert, J.B., de Montleau, C., et al. (2021). Partial recovery of visual function in a blind patient after optogenetic therapy. Nat. Med. 27, 1223-1229.

37. Aurnhammer, C., Haase, M., Muether, N., Hausl, M., Rauschhuber, C., Huber, I., Nitschko, H., Busch, U., Sing, A., Ehrhardt, A., et al. (2012). Universal real-time PCR for the detection and quantification of adeno-associated virus serotype 2-derived inverted terminal repeat sequences. Hum. Gene Ther. Methods 23, 18-28.

38. Sieving, P.A., Murayama, K., and Naarendorp, F. (1994). Push-pull model of the primate photopic electroretinogram: a role for hyperpolarizing neurons in shaping the b-wave. Vis. Neurosci. 11, 519-532.

39. Missel, P.J. (2012). Simulating intravitreal injections in anatomically accurate models for rabbit, monkey, and human eyes. Pharm. Res. 29, 3251-3272. 